\title{
Distribución y diversidad de colémbolos (Hexapoda: Collembola) en el gradiente altitudinal de un bosque templado en México
}

\author{
Arturo García-Gómez ${ }^{1}$, Gabriela Castaño-Meneses ${ }^{1,2}$ \& José G. Palacios-Vargas ${ }^{1}$ \\ 1. Ecología y Sistemática de Microartrópodos, Departamento de Ecología y Recursos Naturales, Universidad Nacional \\ Autónoma de México, Facultad de Ciencias, Ciudad Universitaria, C. P. 04510 México, D.F.; \\ gab12y@yahoo.com.mx,jgpv@hp.fciencias.unam.mx \\ 2. Unidad Multidisciplinaria de Docencia e Investigación, Facultad de Ciencias, Universidad Nacional Autónoma de \\ México, Campus Juriquilla, Boulevard Juriquilla, 3001, C. P. 76230, Querétaro, Querétaro, México; \\ gcm@hp.fciencias.unam.mx
}

Recibido 19-V-2010. Corregido 30-IX-2010. Aceptado 30-X-2010.

\begin{abstract}
Distribution and diversity of springtails (Hexapoda: Collembola) on the altitudinal gradient of a temperate forest in Mexico. Mountain ecosystems have shown slow mineralization activity due to weather conditions, and to some groups with arthropods with special roles. The Collembola is an important group for litter fragmentation, showing different distribution patterns. The objective of the present study was to determine the diversity of Collembola along a volcano altitudinal gradient. For this, four sampling expeditions evaluated four altitudinal levels ( $\mathrm{I}=2$ 753, II=3 015, III=3 250 and IV=3 687 masl) in Iztaccihuatl Volcano, from November 2003, and March, June and August 2004. Shannon diversity (H'), Pielou evenness (J') and Simpson dominance $(1 / \lambda)$ indices were calculated. The similarity between the associations of springtails between the sampling sites was evaluated by a cluster analysis using the Pearson correlation coefficient, as distance and the unpaired arithmetic averages (UPGMA) as amalgamation method. A total of 24075 springtails, distributed in 12 families, 46 genera and 86 species was collected. The higher species abundance was found at the altitudinal area II. The lowest diversity value was recorded in IV, while the higher diversity values were found in III. Significant differences between Shannon indices were found between zones II-III $\left(\mathrm{t}_{0.01,187}=4.11, \mathrm{p}<0.05\right)$ and between III-IV $\left(\mathrm{t}_{0.01,187}=3.8, \mathrm{p}<0.05\right)$ according to modified $t$-test. When considering sampling dates, no significant differences were found. The dendrogram showed that in composition the level I is more homogeneous throughout the year. In conclusion, a statistically significant seasonal variation in springtail abundances was not found, but it was observed that, the lower altitude (I) resulted more homogeneous along the studied period, followed by level II and III. There is a particular assemblage of springtails community in each altitudinal area studied; in general, the sites with low slope resulted more diverse in Collembola communities. These results show that there are important factors such as altitude, vegetation type and microhabitat heterogeneity that may affect the distribution of springtails communities along an altitudinal gradient. Rev. Biol. Trop. 59 (1): 315-327. Epub 2011 March 01.
\end{abstract}

Key word: Volcano Iztaccíhuatl, Collembola, diversity, distribution, altitudinal gradient.

En México, los bosques templados de montaña son ecosistemas que han sido relativamente poco estudiados, en general a lo referente de la mesofauna de sus suelos, y en particular, en lo que involucra a los microartrópodos y su relación con el suelo. Se pueden mencionar algunos trabajos realizados en suelos de la Sierra de Quetzaltepec, Estado de México (Miranda \& Palacios-Vargas 1992) y el
Popocatépetl (Palacios-Vargas 1988); en epífitas en el derrame volcánico del Chichinautzin, Morelos (Palacios-Vargas 1981), El Chico, Hidalgo (Palacios-Vargas \& Castaño-Meneses 2002), y con musgos corticícolas en el Iztaccíhuatl (Cutz-Pool et al. 2008).

Por otro lado, los ecosistemas de montaña son hábitats donde la actividad de mineralización es más lenta debido a las condiciones 
climáticas propias de la altitud, temperatura, precipitación, tipo de vegetación y tipo de suelo, entre otros factores, por lo que el papel de la fauna edáfica es indispensable para acelerar tales procesos (Liu et al. 2000, Thaiutsa \& Granger 2000). De tal forma que, la gran diversidad de organismos reside, principalmente, tanto en la hojarasca como en el suelo (BehanPelletier \& Newton 1999), y se ha sugerido que las diferentes agrupaciones edáficas presentan un potencial como bioindicadores (Linden et al. 1994, van Straalen 1997, 1998).

Dentro de la mesofauna se encuentran los microartrópodos, los que comprenden principalmente ácaros, arácnidos y hexápodos, siendo los oribátidos y colémbolos los grupos dominantes en el suelo y hojarasca de bosques, ya que representan más del $90 \%$ del total de individuos (Ducarme et al. 2004, Jing et al. 2005, Cutz-Pool et al. 2006).

Hopkin (1997) menciona, que los colémbolos presentan patrones de dispersión restringido, principalmente por no presentar alas y por su tamaño pequeño $(0.25 \mathrm{~mm}-2 \mathrm{~mm})$; sin embargo, se ha visto que por presentar un tamaño y una masa mínima, se han distribuido por todos los ecosistemas alrededor del mundo, ya sea por medios aéreos o acuáticos (Hawes et al. 2007). Así mismo se ha encontrado que factores como la humedad ambiental (Christiansen 1992), temperatura, pH (Schowalter 2006) y $\mathrm{CO}_{2}$ (García-Gómez et al. 2009), entre otros, son determinantes para el establecimiento de las comunidades de colémbolos.

El presente trabajo se llevó a cabo con la finalidad de determinar la diversidad de comunidades de colémbolos edáficos del Volcán Iztaccíhuatl, México, a lo largo de un gradiente altitudinal. En general, se ha mencionado la existencia de tendencias sobre un decremento de la diversidad de especies con respecto a la altitud, principalmente en plantas, mamíferos y algunos hexápodos, como hormigas (Pianka 1966, Heaney 2001, O’Donnell \& Kumar 2006). Sin embargo, otros grupos taxonómicos, como los microartrópodos, pueden mostrar patrones diferentes (Stevens 1992). Nuestra hipótesis es que, en niveles altitudinales intermedios, las condiciones del bosque templado favorecen la diversidad de especies de colémbolos, mientras que en los límites superiores, la condiciones resultan adversas, registrando un decremento en la riqueza de especies, tal como lo mencionan Liu et al. (2000). Dadas las características de cada piso altitudinal, se espera que las asociaciones de colémbolos sean particulares para cada altitud.

\section{MATERIAL Y MÉTODOS}

Área de estudio: El presente trabajo se realizó en la ladera noroeste del Volcán Iztaccíhuatl (19¹0'20" N-98³8'30" W), localizado a $64 \mathrm{~km}$ al SE de la Ciudad de México, al extremo sur de la Sierra Nevada. La zona de estudio abarcó desde los 2750 hasta los 3680m.s.n.m.; el tipo de vegetación en el gradiente estudiado se describe en el Cuadro 1.

Se llevaron a cabo cuatro muestreos, dos en época de secas (noviembre del 2003, marzo del 2004) y dos en lluvias (junio y agosto del 2004), en cuatro pisos altitudinales (Cuadro 1). Se realizó un transecto de $20 \times 1 \mathrm{~m}^{2}$, marcando al azar diez puntos, donde se extrajeron diez muestras de hojarasca y diez de suelo, haciendo un total de 320 muestras en todo el estudio.

Las muestras se procesaron en el laboratorio, colocándose en embudos de Berlese-Tullgren durante seis días. Los colémbolos fueron separados bajo el microscopio estereoscópico, realizando preparaciones semipermanentes en líquido de Hoyer para su identificación bajo el microscópico de contraste de fases.

Se calcularon los índices de diversidad de Shanon (H'), equitatividad de Pielou (J'), y de dominancia de Simpson $(1 / \lambda$, también se puede denotar como 1/D), de acuerdo con Ludwing \& Reynolds (1988). Los índices de diversidad se compararon utilizando una prueba de $t$ de student modificada (Magurran 1988, Zar 1984), utilizando la corrección de Bonferroni para comparaciones múltiples (altitudes $\alpha=0.05 / 4=0.01$, fechas $\alpha=0.05 / 4=0.01$ ). Para ver la similitud entre las asociaciones de colémbolos en las diferentes épocas de recolección y las altitudes, se efectúo un análisis de cluster, utilizando el 


\section{CUADRO 1}

Características generales de los puntos de colecta a lo largo de un gradiente altitudinal en el Volcán Iztaccíhuatl, Estado de México, México

TABLE 1

General information of sampling points along an altitudinal gradient in Iztaccihuatl volcano, Mexico State, Mexico

\begin{tabular}{|c|c|c|c|c|c|}
\hline Zona & $\begin{array}{l}\text { Altitud } \\
\text { (m) }\end{array}$ & Coordenadas & Pendiente & Tipo de bosque & Vegetación dominante \\
\hline I & 2753 & $\begin{array}{l}19^{\circ} 12^{\prime} 40^{\prime \prime} \mathrm{N} \\
98^{\circ} 44^{\prime} 2^{\prime \prime} \mathrm{W}\end{array}$ & $29^{\circ}$ & Bosque de encino & $\begin{array}{l}\text { Quercus laurina, Budleia cordata, Pinus } \\
\text { sp., Cupressus sp., Smilax sp. Cucurbita sp., } \\
\text { Berberis moranensis, Viburnum stenocalyx y } \\
\text { Garrya laurifolia }\end{array}$ \\
\hline II & 3015 & $\begin{array}{l}19^{\circ} 12^{\prime} 30^{\prime \prime} \mathrm{N} \\
98^{\circ} 43^{\prime} 41^{\prime \prime} \mathrm{W}\end{array}$ & $36^{\circ}$ & Bosque de encino & $\begin{array}{l}\text { Quercus laurina, Q. magnolifolia, Pinus sp., } \\
\text { Cupressus sp. y Smilax sp. }\end{array}$ \\
\hline III & 3250 & $\begin{array}{l}19^{\circ} 12^{\prime} 16^{\prime \prime} \mathrm{N} \\
98^{\circ} 42^{\prime} 54^{\prime \prime} \mathrm{W}\end{array}$ & $33^{\circ}$ & $\begin{array}{l}\text { Bosque de Abies con } \\
\text { pocos pinos }\end{array}$ & $\begin{array}{l}\text { Abies religiosa, Pinus sp., Cupressus lindeyi, } \\
\text { Rodaldana sp., Senecio sp. y Habenaria sp. }\end{array}$ \\
\hline IV & 3687 & $\begin{array}{l}19^{\circ} 12^{\prime} 66^{\prime \prime} \mathrm{N} \\
98^{\circ} 41^{\prime} 25^{\prime \prime} \mathrm{W}\end{array}$ & $35^{\circ}$ & $\begin{array}{l}\text { Bosque de pino con } \\
\text { zacatonal }\end{array}$ & $\begin{array}{l}\text { Pinus hartwegii, Lupinus montanus y Acyrium } \\
\text { hypericoides }\end{array}$ \\
\hline
\end{tabular}

coeficiente de correlación de Pearson como distancia, y como método de amalgama las medias aritméticas no pareadas (UPGMA; Pearson 1977, Sokal \& Michener 1958)

\section{RESULTADOS}

Durante las cuatro fechas de recolección se obtuvieron 77113 organismos, de los que el
$31.22 \%$ (24 075) pertenecen a los colémbolos, mostrando una densidad de 59461 ind. $/ \mathrm{m}^{2}$.

Se encontraron 86 especies distribuidas en 12 familias y 46 géneros (Cuadro 2), las tres familias más abundantes son: Isotomidae (40.8\%), Hypogastruridae (19.9\%) y Onychiuridae (11.9\%; Fig. 1).

Las familias con mayores densidades en época de lluvias son: Hypogastruridae,

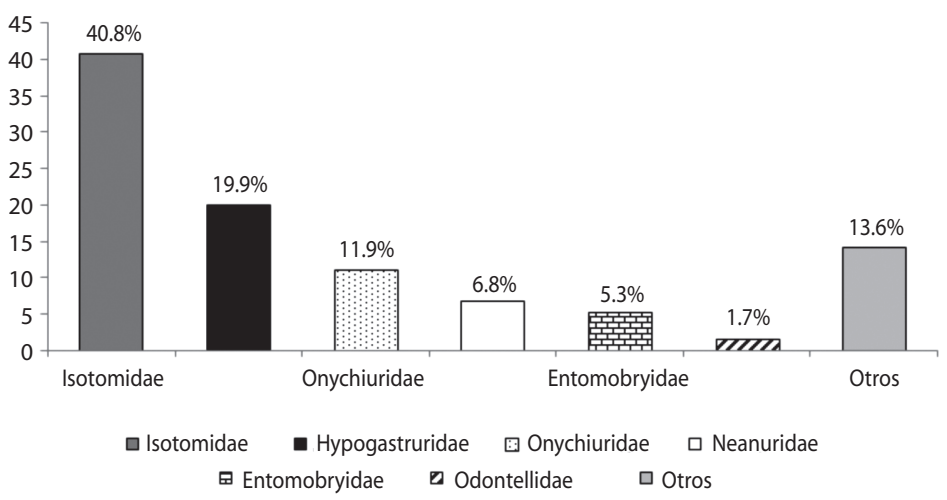

Fig. 1. Abundancia relativa de familias de Collembola encontradas en la ladera noroeste del Volcán Iztaccíhuatl, Estado de México, México. Otros corresponden a Tomoceridae, Sminthurididae, Katiannidae, Dicyrtomidae, Sminthuridae y Neelidae. Fig. 1. Relative abundance of Collembola families found in the Northwestern slope of Iztaccíhuatl volcano, State of Mexico, Mexico. As others we included: Tomoceridae, Sminthurididae, Katiannidae, Dicyrtomidae, Sminthuridae and Neelidae. 
CUADRO 2

Lista taxonómica de la comunidad de colémbolos en cuatro altitudes durante cuatro fechas de recolecta, de la ladera noroeste del Volcán Iztaccíhuatl, Estado de México, México

TABLE 2

Taxonomic list of the springtails community by altitudinal areas and collection date from the Northwest slope of the Iztaccihuatl volcano, Mexico State, Mexico

\begin{tabular}{|c|c|c|c|c|c|c|c|c|}
\hline $\begin{array}{c}\text { Especies } \backslash \text { Localidad (msnm) - } \\
\text { Fecha de colecta }\end{array}$ & $\begin{array}{c}\text { I } \\
2753\end{array}$ & $\begin{array}{c}\text { II } \\
3015\end{array}$ & $\begin{array}{c}\text { III } \\
3250\end{array}$ & $\begin{array}{c}\text { IV } \\
3687\end{array}$ & Nov. & Mar. & Jun. & Ago. \\
\hline
\end{tabular}

Hypogastruridae

Hypogastrura ca. hispanica

Hypogastrura ca. sensilis

Ceratophysella ca. brevis

Ceratophysella ca. denticulata

Ceratophysella ca. succinea

Schoettella ca. glasgowi

Willemia iztaccihuatlensis

Stenogastrura sp.

Odontellidae

Odontella ca. denticulada

Superodontella ca. conglobata

Superodontella ca. nana

Stachiomella sp.

Neanuridae

Friesea hoffmannorum

Neanura muscorum

Americanura ca. prima

Americanura ca. izabalana

Pseudachorutes ca. romeroi

Pseudachorutes ca. simplex

Micranurida pygmaea

Onychiuridae

Protaphorura macrodentata

Protaphorura meridiana

Mesaphorura florae

Mesaphorura krausbaueri

Mesaphorura macrochaeta

Mesaphorura yosiii

Metaphorura affinis

Fissuraphorura sp.

Isotomidae

Folsomides chichinautzini

Proisotoma ca. alticola

Proisotoma frisoni

Proisotoma minuta

Proisotoma ca. sepulcralis ${ }^{1}$

Proisotoma ca. sepulcralis ${ }^{2}$

Proisotoma sp. 1 
CUADRO 2 (Continuación)

Lista taxonómica de la comunidad de colémbolos en cuatro altitudes durante cuatro fechas de recolecta, de la ladera noroeste del Volcán Iztaccíhuatl, Estado de México, México

TABLE 2 (Continued)

Taxonomic list of the springtails community by altitudinal areas and collection date from the Northwest slope of the Iztaccihuatl volcano, Mexico State, Mexico

\begin{tabular}{|c|c|c|c|c|c|c|c|c|}
\hline $\begin{array}{c}\text { Especies } \backslash \text { Localidad (msnm) - } \\
\text { Fecha de colecta }\end{array}$ & $\begin{array}{c}\text { I } \\
2753\end{array}$ & $\begin{array}{l}\text { II } \\
3015\end{array}$ & $\begin{array}{l}\text { III } \\
3250\end{array}$ & $\begin{array}{l}\text { IV } \\
3687\end{array}$ & Nov. & Mar. & Jun. & Ago. \\
\hline Proisotoma sp. 2 & & & $*$ & $*$ & $*$ & $*$ & & \\
\hline Proisotoma sp. 3 & & $*$ & $*$ & & $*$ & $*$ & & \\
\hline Proisotoma sp. 4 & & & & $*$ & $*$ & $*$ & & \\
\hline Ballistura ca. obtusicauda & $*$ & & & & $*$ & & & \\
\hline Folsomina onychiurina & $*$ & $*$ & $*$ & & $*$ & $*$ & $*$ & $*$ \\
\hline Clavisotoma ca. plicicauda & $*$ & $*$ & $*$ & & $*$ & $*$ & $*$ & $*$ \\
\hline Hemisotoma thermophila & $*$ & & * & $*$ & $*$ & & * & $*$ \\
\hline Isotomurus ca. palustroides & & & & * & & & $*$ & \\
\hline Isotomorus ca. tricolor & $*$ & $*$ & $*$ & $*$ & $*$ & & $*$ & $*$ \\
\hline Isotomiella minor & * & $*$ & $*$ & & $*$ & $*$ & $*$ & $*$ \\
\hline Isotoma ca. subviridis & $*$ & $*$ & $*$ & * & $*$ & $*$ & $*$ & $*$ \\
\hline Parisotoma ca. notabilis & $*$ & $*$ & $*$ & $*$ & $*$ & $*$ & $*$ & $*$ \\
\hline Parisotoma ca. tariva & $*$ & $*$ & $*$ & $*$ & $*$ & $*$ & $*$ & $*$ \\
\hline Desoria ca. flora & & & & $*$ & * & $*$ & $*$ & $*$ \\
\hline Granisotoma comuna & & & & $*$ & & & & $*$ \\
\hline \multicolumn{9}{|l|}{ Entomobryidae } \\
\hline Orchesella ca. folsomi & $*$ & $*$ & $*$ & & & $*$ & & $*$ \\
\hline Americabrya arida & * & $*$ & * & * & $*$ & $*$ & * & $*$ \\
\hline Entomobrya ca. triangularis & $*$ & $*$ & $*$ & $*$ & $*$ & $*$ & $*$ & $*$ \\
\hline Entomobrya sp. 1 & & $*$ & $*$ & & $*$ & $*$ & & \\
\hline Entomobrya sp. 2 & $*$ & $*$ & $*$ & $*$ & $*$ & $*$ & & \\
\hline Entomobrya sp. 3 & & $*$ & $*$ & & $*$ & $*$ & $*$ & $*$ \\
\hline Willowsia mexicana & $*$ & $*$ & * & $*$ & $*$ & $*$ & * & $*$ \\
\hline Willowsia ca. nigromaculata & $*$ & & & $*$ & & * & & \\
\hline Seira purpurea & & $*$ & & * & $*$ & $*$ & & \\
\hline Lepidocyrtus finus & & $*$ & $*$ & $*$ & $*$ & $*$ & $*$ & $*$ \\
\hline Pseudosinella ca. dubia & & $*$ & $*$ & $*$ & & $*$ & $*$ & $*$ \\
\hline Pseudosinella ca. hirsuta & & & * & & $*$ & & & \\
\hline Pseudosinella ca. orba & & & $*$ & & $*$ & & & \\
\hline Pseudosinella ca. sexoculata & & $*$ & $*$ & & $*$ & $*$ & $*$ & $*$ \\
\hline Pseudosinella ca. testa & & & & $*$ & * & & & \\
\hline \multicolumn{9}{|l|}{ Tomoceridae } \\
\hline Plutomurus ca. californicus & $*$ & & & & & $*$ & & $*$ \\
\hline \multicolumn{9}{|l|}{ Sminthurididae } \\
\hline Sminthurides ca. lepus & & $*$ & $*$ & $*$ & & & & $*$ \\
\hline Sminthurides ca. occultus & $*$ & $*$ & $*$ & $*$ & $*$ & & & $*$ \\
\hline Sminthurides sp. & $*$ & $*$ & $*$ & $*$ & $*$ & & $*$ & $*$ \\
\hline Sphaeridia pumilis & $*$ & $*$ & * & $*$ & * & & * & $*$ \\
\hline \multicolumn{9}{|l|}{ Katiannidae } \\
\hline Polykatianna ca. intermedia & & $*$ & $*$ & $*$ & $*$ & $*$ & $*$ & \\
\hline
\end{tabular}


CUADRO 2 (Continuación)

Lista taxonómica de la comunidad de colémbolos en cuatro altitudes durante cuatro fechas de recolecta, de la ladera noroeste del Volcán Iztaccíhuatl, Estado de México, México

TABLE 2 (Continued)

Taxonomic list of the springtails community by altitudinal areas and collection date from the Northwest slope of the Iztaccihuatl volcano, Mexico State, Mexico

\begin{tabular}{|c|c|c|c|c|c|c|c|c|}
\hline $\begin{array}{c}\text { Especies } \backslash \text { Localidad (msnm) - } \\
\text { Fecha de colecta }\end{array}$ & $\begin{array}{c}\mathrm{I} \\
2753\end{array}$ & $\begin{array}{c}\text { II } \\
3015\end{array}$ & $\begin{array}{c}\text { III } \\
3250\end{array}$ & $\begin{array}{c}\text { IV } \\
3687\end{array}$ & Nov. & Mar. & Jun. & Ago. \\
\hline Sminthurinus ca. elegans & $*$ & & & $*$ & & $*$ & $*$ & $*$ \\
\hline Sminthurinus ca. quadrimaculatus & $*$ & $*$ & $*$ & $*$ & & & $*$ & \\
\hline Sminthurinus sp. & & & & $*$ & & & $*$ & \\
\hline \multicolumn{9}{|l|}{ Dicyrtomidae } \\
\hline Dicyrtoma ca. mitra & & $*$ & & & & & $*$ & \\
\hline Ptenothrix ca. atra & $*$ & & $*$ & & & & $*$ & $*$ \\
\hline Ptenothrix ca. californica & & $*$ & $*$ & $*$ & & & $*$ & $*$ \\
\hline Ptenothrix ca. renateae & $*$ & $*$ & * & $*$ & & & * & $*$ \\
\hline Ptenothrix ca. texensis & & & $*$ & & & & $*$ & $*$ \\
\hline \multicolumn{9}{|l|}{ Sminthuridae } \\
\hline Sminthurus ca. eisenii & $*$ & * & $*$ & * & $*$ & $*$ & $*$ & $*$ \\
\hline Sminthurus ca. incisus & $*$ & $*$ & & & * & & $*$ & $*$ \\
\hline Sminthurus ca. fitchi & & & & $*$ & & $*$ & & \\
\hline Sminthurus ca. sagittus & $*$ & & & $*$ & $*$ & $*$ & & \\
\hline \multicolumn{9}{|l|}{ Neelidae } \\
\hline Neelus murinus & $*$ & $*$ & $*$ & & $*$ & & & $*$ \\
\hline Neelides minutus & $*$ & $*$ & $*$ & & $*$ & $*$ & $*$ & $*$ \\
\hline Megalothorax incertus & $*$ & $*$ & $*$ & & & $*$ & $*$ & $*$ \\
\hline Megalothorax minimus & $*$ & $*$ & $*$ & $*$ & $*$ & $*$ & $*$ & $*$ \\
\hline S & 45 & 58 & 57 & 49 & 56 & 48 & 55 & 55 \\
\hline $\mathrm{H}^{\prime}$ & 2.43 & 1.98 & 2.88 & 2.11 & 3.05 & 2.78 & 2.52 & 2.74 \\
\hline$J^{\prime}$ & 0.68 & 0.48 & 0.71 & 0.54 & 0.75 & 0.72 & 0.63 & 0.68 \\
\hline$\lambda$ & 0.15 & 0.32 & 0.09 & 0.24 & 0.07 & 0.096 & 0.15 & 0.12 \\
\hline N1 & 11.3 & 7.27 & 17.88 & 8.33 & 21.1 & 16.14 & 12.5 & 15.63 \\
\hline $\mathrm{N} 2$ & 6.51 & 3.11 & 10.14 & 4.04 & 13.6 & 10.34 & 6.34 & 7.9 \\
\hline
\end{tabular}

Riqueza (S) diversidad (H'), equitatividad (J'), dominancia ( $\lambda$ ), número de especies abundantes (N1) y muy abundantes (N2). Se encontraron dos especies cercanas a Proisotoma sepulcralis, las cuales se asignan con los índices, 1 y 2.

Species richness (S) diversity (H'), evenness (J'), dominancy $(\lambda)$, number of abundant (N1) and very abundant species (N2). Two species close to Proisotoma sepulcralis, were found, which are indicated with the indices, 1 and 2.

Tomoceridae, Sminthurididae, Dicyrtomidae y Sminthuridae, y en secas Odontellidae, Neanuridae, Onychiuridae, Entomobryidae y Neelidae; en el caso de Isotomidae y Katiannidae presentan una distribución similar a lo largo del año (Fig. 2).
Espacialmente, Onychiuridae, Tomoceridae, Katianidae, Dicyrtomidae y Sminthuridae, prefieren el primer piso para su establecimiento; Hypogastruridae y Odontellidae presentan una mayor distribución en el piso II; en el piso III se encuentran en mayor número de 


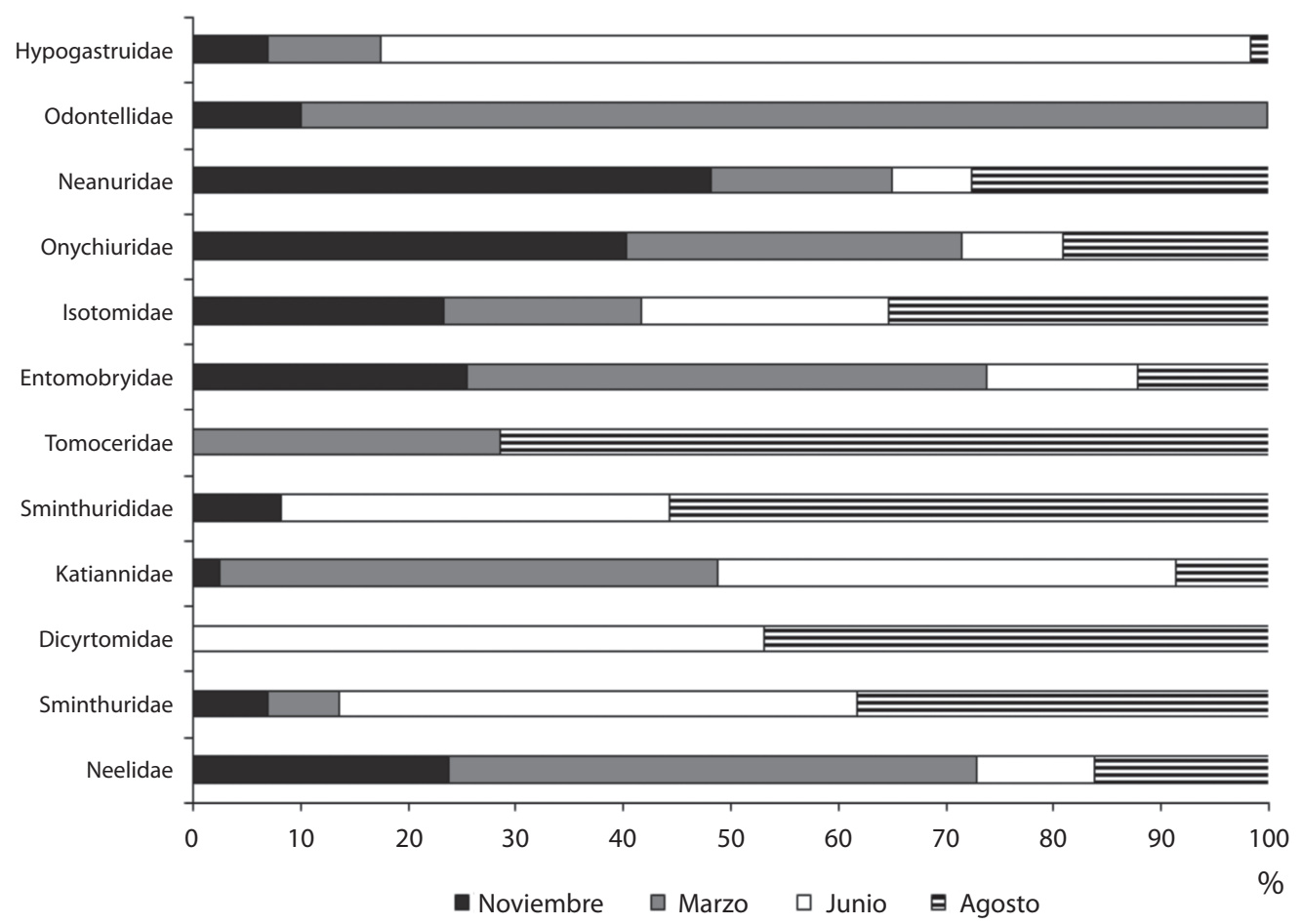

Fig. 2. Abundancia relativa de familias de colémbolos por fecha de recolecta del Volcán Iztaccíhuatl, México.

Fig. 2. Relative abundance of springtail families in each collection date in Iztaccíhuatl Volcano, Mexico.

Entomobryidae y Neelidae; la distribución de Neanuridae es mayor en el piso IV, e Isotomidae y Sminthurididae no tienen algún piso de preferencia, es decir, se distribuyen de forma similar a lo largo del gradiente altitudinal (Fig. 3).

En las altitudes, el piso II presenta la menor diversidad de organismos, siendo el III el más diverso, tanto en los valores del índice de Shannon como el de Simpson; sin embargo, la mayor equitatividad se encuentra en las zonas I y III (Cuadro 2). Además, se hallaron diferencias significativas entre los índices de diversidad de Shannon registrados en los pisos II-III $\left(t_{0.01,187}=4.11, \mathrm{p}<0.05\right)$ y entre III-IV $\left(t_{0.01,187}=3.8, \mathrm{p}<0.05\right)$.

En las diferentes fechas de recolección, noviembre, junio y agosto presentan la mayor diversidad, y marzo la menor riqueza de especies. Con relación a los índices de Shannon, noviembre presentó la mayor diversidad y junio la menor y de acuerdo con el índice de Simpson, junio fue el más diverso (Cuadro 2). Por otro lado, entre las diferentes fechas de recolección, no se encontraron diferencias significativas entre los valores registrados para los índices de diversidad de Shannon $\left(t_{0.01}\right.$, ${ }_{187}=2.71, \mathrm{p}<0.05$ ).

En cada piso altitudinal, con relación a las fechas de recolección, se encontró el piso III con los índices de diversidad más altos, tanto en la época de secas, como en lluvias. En noviembre, marzo y agosto, el piso IV presenta la diversidad más baja. En junio el piso II es el que presenta los índices bajos (Cuadro 3).

En tres de las cuatro fechas hay diferencias significativas entre los distintos pisos; en noviembre y agosto, éstas se presentaron entre 


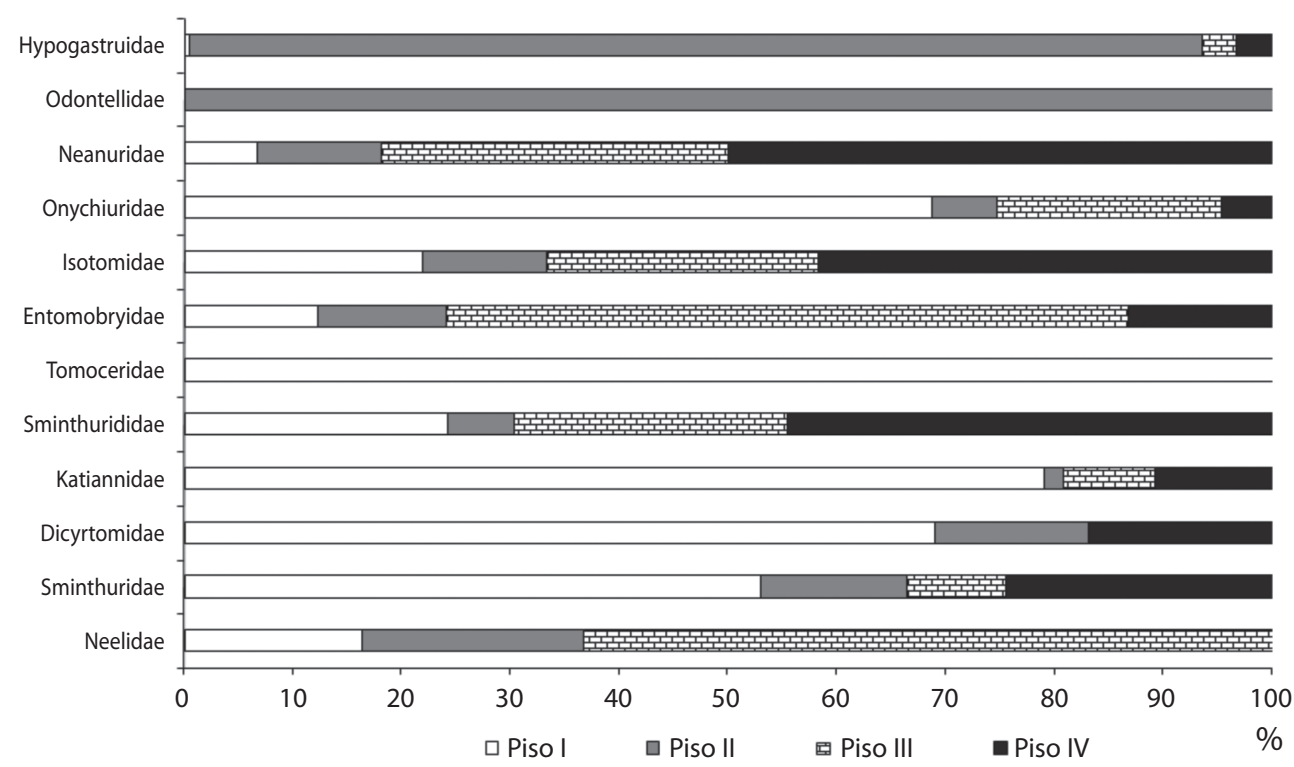

Fig. 3. Abundancia relativa de familias de colémbolos en cuatro pisos altitudinales de la ladera noroeste del volcán Iztaccíhuatl, Estado de México, México.

Fig. 3. Relative abundance of springtail families in four altitudinal levels in the Northwest slope of the Iztaccihuatl volcano, Mexico State, Mexico.

\section{CUADRO 3}

Índice de diversidad, por pisos altitudinales en cada fecha de colecta, de colémbolos de la ladera noroeste del Volcán Iztaccíhuatl, México

TABLE 3

Diversity index of springtails by altitudinal areas and collection date from Northwest slope of the Iztaccihuatl volcano, Mexico

\begin{tabular}{ccccccccccccccccccc} 
Fecha & \multicolumn{4}{c}{ Noviembre } & \multicolumn{1}{c}{ Marzo } & \multicolumn{4}{c}{ Junio } & \multicolumn{4}{c}{ Agosto } \\
& I & II & III & IV & I & II & III & IV & I & II & III & IV & I & II & III & IV \\
S & 28 & 34 & 34 & 26 & 23 & 29 & 25 & 15 & 25 & 31 & 28 & 24 & 28 & 27 & 35 & 31 \\
H' & 2.1 & 2.6 & 2.8 & 2.1 & 2.2 & 2.25 & 2.3 & 1.9 & 2.2 & 1.05 & 2.3 & 1.9 & 2.1 & 2.3 & 2.6 & 1.9 \\
J' & 0.6 & 0.75 & 0.79 & 0.6 & 0.7 & 0.6 & 0.72 & 0.7 & 0.68 & 0.3 & 0.7 & 0.6 & 0.63 & 0.71 & 0.75 & 0.5 \\
口 & 0.2 & 0.1 & 0.08 & 0.1 & 0.18 & 0.17 & 0.13 & 0.21 & 0.14 & 0.6 & 0.13 & 0.2 & 0.22 & 0.18 & 0.1 & 0.27 \\
N1 & 8.2 & 14.2 & 16.5 & 8.8 & 9.0 & 9.4 & 10.3 & 6.7 & 9.1 & 2.8 & 10.7 & 6.9 & 8.3 & 10.4 & 14.8 & 6.8 \\
N2 & 4.5 & 9.9 & 11.8 & 5.2 & 5.3 & 5.8 & 7.3 & 4.6 & 6.7 & 1.6 & 7.3 & 3.6 & 4.4 & 5.5 & 8.9 & 3.6
\end{tabular}

Abreviaturas como en el Cuadro 2.

Abbreviations as in Table 2. 
el I-III y III-IV, en junio entre I-II, II-III y II-IV; sólo marzo no presentó diferencias significativas (Cuadro 4).

El dendrograma obtenido por medio del análisis de cluster (Fig. 4), muestra que el piso I es más homogéneo en su composición a lo largo del año; los pisos II y III se asocian de forma similar en cada fecha de recolección, y la última altitud presenta una misma agrupación, exceptuando agosto, dónde presenta una semejanza más cercana a los pisos intermedios.

\section{DISCUSIÓN}

Al término de las colectas, se encontró gran abundancia de colémbolos tanto espacial

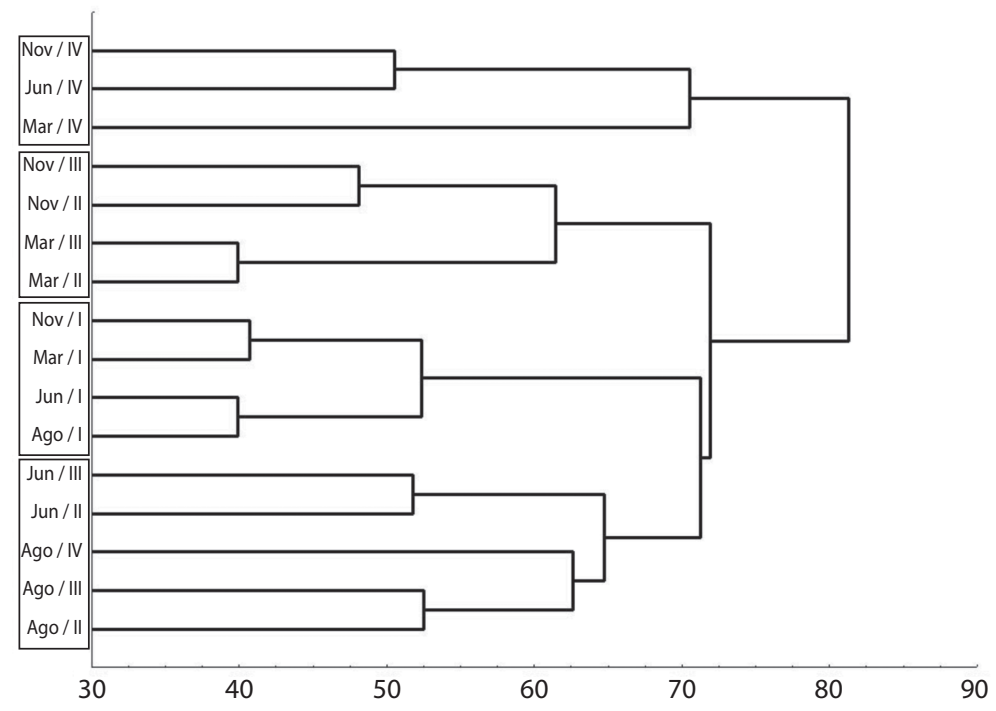

Fig. 4. Dendrograma de las asociaciones de especies de colémbolos edáficos de cuatro pisos altitudinales en cuatro fechas de recolecta, en el volcán Iztaccíhuatl, Estado de México, México.

Fig. 4. Dendrogram of the relationship between the associations of Collembola species from soil in four altitudes at four collection dates from Iztaccíhuatl volcano, Mexico State, Mexico.

\section{CUADRO 4}

Valor de t para la comparación entre los índices de diversidad de cuatro pisos altitudinales de la ladera noroeste del Volcán Iztaccíhuatl, México

TABLE 4

$t$ value to compare between rates of four altitudinal diversity of the Northwest slope of the volcano Iztaccihuatl, Mexico

\begin{tabular}{ccccccccccccccccc} 
Recolectas & \multicolumn{3}{c}{ Noviembre } & \multicolumn{1}{c}{ Marzo } & \multicolumn{4}{c}{ Junio } & \multicolumn{4}{c}{ Agosto } \\
Piso & I & II & III & IV & I & II & III & IV & I & II & III & IV & I & II & III & IV \\
I & - & 3 & $* 3.9$ & 0.33 & - & 0.2 & 0.8 & 1.7 & - & $* 6.1$ & 1.1 & 1.5 & - & 1.2 & $* 3.2$ & 0.1 \\
II & & - & 1.1 & 2.84 & & - & 0.5 & 2.1 & & - & $* 6.8$ & $* 4.1$ & & - & 2.1 & 2.2 \\
III & & & - & $* 3.9$ & & & - & 2.7 & & & - & 0.1 & & - & $* 4.2$ \\
IV & & & & - & & & & - & & & & - & & & -
\end{tabular}

$\left({ }^{*} \mathrm{p}>0.05\right)$, g.l.=3.02, con corrección de Bonferroni. $\left({ }^{*} \mathrm{p}>0.05\right)$, g.l=3.02, with Bonferroni correction. Abreviaturas como en el Cuadro 2. Abbreviations as in Table 2. 
como temporalmente, comparado con el trabajo de Cutz-Pool et al. (2005), mismo que se enfoca a los que viven en musgos corticícolas. Se halló un mayor número de familias, ya que el hábitat edáfico (suelo y hojarasca en conjunto) conforma un sitio donde las fluctuaciones ambientales de temperatura y humedad son más estables (Schowalter 2006) y, por consiguiente ideal para una mayor diversidad de organismos.

Con relación al trabajo realizado en el Popocatépetl (macizo más próximo) por Palacios-Vargas (1985), encontramos el mismo número de familias, pero un aumento considerable de géneros (47 vs. 28) y especies (86 vs. 38).

Tales diferencias puede deberse a la edad geológica de los dos volcanes, ya que al ser más antiguo el Volcán Iztaccíhuatl (Barrera 1968), puede presentar ciertas especies relictas, o en su caso, especies que han evolucionado con presiones ambientales diferentes a las encontradas en el Popocatépetl, a pesar de presentar condiciones altitudinales semejantes.

Hasegawa et al. (2006), encontraron que la composición de oribátidos se ve afectada conforme se aumenta la altitud, de igual forma ocurre con los colémbolos del Iztaccíhuatl, los que presentan un conjunto de especies particulares, en cada piso altitudinal.

Entre las especies encontradas, Desoria ca. flora, por su presencia durante todo el año, podría emplearse como indicador de altitud, principalmente del piso IV. Otro ejemplo sería la familia Odontellidae, cuyos representantes se les halló exclusivamente en el piso II, aunque por el deterioro que ha sufrido el bosque, pudo haber afectado la distribución de toda la familia.

Además, encontramos especies temporales, por ejemplo Ceratophysella ca. brevis, encontrada en todos los pisos altitudinales, pero sólo en época de lluvias.

También se registraron especies en tres de las cuatro altitudes, y no las encontramos en el piso II o en el III, llegando a concluir que en dichos pisos las condiciones bióticas, abióticas o interacción de ambas evitan su establecimiento.

En el caso de la diversidad, Rusek (1998) y O’Donell \& Kumar (2006), mencionan que la composición de organismos aumenta conforme la humedad se incrementa, sin embargo, en el presente trabajo no ocurre lo mencionado, por lo menos en lo referente a la temporalidad. Esto puede deberse a las diferentes condiciones de altitud y tipo de vegetación, que pueden dar como consecuencia una heterogeneidad donde se crean microambientes cuyas variables abióticas, como temperatura e insolación, entre otras, pueden mantenerse constantes a lo largo de las diferentes épocas.

Al analizar las fechas de recolección, se observó que en noviembre, y a pesar de dar inicio la época de secas, se encuentra una gran diversidad, lo que indica que los bosques templados, como el Iztaccíhuatl retienen por mucho más tiempo la humedad ambiental, principalmente en suelo y hojarasca, de tal forma que la humedad promedio es prácticamente la misma tanto en julio como en agosto.

Otro punto importante es la capa de hojarasca que se encuentra en los diferentes puntos, y a pesar de no haber medido su profundidad, podemos inferir su importancia en este aspecto, ya que Cutz-Pool et al. (2008) encontraron una pérdida de humedad considerable en los musgos corticícolas, de tal forma que existe una migración vertical, de algunas especies, para protegerse de las inclemencias ambientales, como pueden ser la insolación y la pérdida de humedad.

Con relación del efecto de la altitud sobre los colémbolos, hay diferentes resultados en cada piso, encontrando en el piso III la mayor diversidad, la que comparte elementos con el piso I, y presenta diferencias significativas para los pisos II y IV. Estos resultados se deben principalmente a la baja dominancia, en los últimos pisos mencionados, en relación al tercero.

Observando con más detalle las diferentes áreas, se encontró que los sitios más diversos son aquellos de pendientes con menos inclinación, Cassagne et al. (2004) encontraron lo mismo en los Pirineos franceses, donde el 
establecimiento y diversidad de colémbolos es mayor en sitios con pendientes poco pronunciadas; por consiguiente, el relieve, particularmente en este aspecto, es un buen indicador para la distribución de los colémbolos (Jensen et al. 1973), jugando un papel importante para la diversidad de organismos.

El piso altitudinal III, es importante para la diversidad de colémbolos, ya que en las cuatro fechas de recolección siempre se presentó con el de mayor índice. El piso que le sigue en este aspecto, es el II (excepto en junio), donde se presentan los mayores grados de inclinación, y por ende el lavado constante de nutrimentos, además de la pérdida de microartrópodos, debido a la acción mecánica del agua, por consiguiente es de considerar a la hojarasca como una variable importante, para la distribución de los colémbolos (Badejo et al. 1998, Hou et al. 2005). Además, esta variable es diferente en cada zona por el tipo de bosque, así como por el aumento de la capa de hojarasca, donde su incremento se ve relacionado directamente con el aumento humedad y de organismos (Irmler 2006).

En el caso del piso IV, se presenta una vegetación alpina, es decir los pinos ya se encuentran más dispersos y los zacatonales comienzan a dominar el paisaje, y la diversidad es menor en este sitio, por consiguiente, las especies que se adaptaran favorablemente a un clima más agreste serán pocas y sólo algunas presentarán grandes densidades poblacionales, como es el caso de Desoria ca. flora (GarcíaGómez et al. 2009).

Desde el punto de vista edafológico, los nutrimentos de cada tipo de vegetación, por la acción de la degradación de las hojas, son distintos principalmente para el nitrógeno, carbono y lignina, entre otros componentes (Scout \& Binkey 1997). De igual forma, estas diferencias se pueden encontrar en un misma vegetación por causa de la acción de la pendiente (Sariyildiz et al. 2005), por consiguiente, la diversidad y abundancia de organismos también seguirá este comportamiento.

En el caso de la composición entre las diferentes zonas encontramos, que el piso I es la más homogénea a lo largo del año, los pisos intermedios presentan una composición similar en fecha de recolección, siendo más estrecha esta relación en lluvias, y como se ha comentado anteriormente, este factor abiótico es de suma importancia principalmente para el establecimiento de organismos. Por otro lado, Koleff et al. (2003) y Prinzing (2005), mencionan la importancia de la heterogeneidad del hábitat es importante para la distribución de los colémbolos. En nuestro estudio, el piso IV muestra una agregación parecida a lo largo de todo el año, excepto en agosto, que resultó el mes más húmedo. En este sentido, podemos mencionar que los Collembola cambian de distribución o abundancia relativa, en respuesta a condiciones del hábitat, a las condiciones microclimáticas de los microhábitats y de la características ecológicas de las mismas especies (André 1983, Prinzing \& Woas 2003, Prinzing 2005).

A modo de conclusiones, se encontraron más especies y géneros que en sitios cercanos, como el Popocatépetl, o en musgos corticícolas de la misma zona, además de organismos cuya distribución es específica a alguna zona en particular.

En el piso II, se encontró la mayor diversidad y equidad; sin embargo, a lo largo del año de recolección no se encontraron diferencias significativas, pero particularmente y exceptuando el mes más húmedo se encontraron diferencias entre los diferentes pisos, viendo de esta forma que la altitud y la heterogeneidad de la ladera es importante para el establecimiento y distribución de los diferentes organismos, y en secas son sitios de condiciones microambientales ideales para la asociación de colémbolos.

\section{AGRADECIMIENTOS}

Leopoldo Cutz, Aldo Bernal, Carmen Maldonado, Ricardo Iglesias y Leonardo González ayudaron en el trabajo de campo; el Consejo Nacional de Ciencia y Tecnología (México) otorgó una beca de posgrado a AGG. 


\section{RESUMEN}

Los colémbolos son fragmentadores importantes de la hojarasca, presentan diferentes patrones de distribución a lo largo de un gradiente altitudinal. El objetivo del presente trabajo es determinar la diversidad y distribución de Collembola en el volcán Iztaccíhuatl. Para ello, se realizaron cuatro muestreos, a lo largo de un año en cuatro pisos altitudinales $(\mathrm{I}=2753$, II=3 015, III=3 250 y $\mathrm{IV}=3$ 687m.s.n.m.) y se calcularon los índices de diversidad de Shannon, equitatividad de Pielou y dominancia de Simpson, para compararlos mediante una prueba de $t$ modificada. Los resultados muestran al piso III como el más diverso, y la mayor equitatividad se encuentra en el II. Además, se hallaron diferencias significativas entre los pisos II-III $\left(t_{0.01,187}=4.11, \mathrm{p}<0.05\right)$ y entre III-IV $\left(t_{0.01}\right.$, $\left.{ }_{187}=3.8, \mathrm{p}<0.05\right)$. Para las fechas de recolección no obtuvimos diferencias significativas $\left(t_{0.01,187}=2.71, \mathrm{p}<0.05\right)$. Al final encontramos que la temporalidad no incrementa la diversidad (estadísticamente), por consiguiente, condiciones como la altitud, vegetación y heterogeneidad geográfica crean microambientes, para protegerse de inclemencias ambientales. El piso I es el más homogéneo, seguida por el II y el III, además hallamos que los sitios más diversos son aquellos de pendientes menos pronunciadas.

Palabras clave: Volcán Iztaccíhuatl, Collembola, diversidad, distribución, gradiente altitudinal.

\section{REFERENCIAS}

André, H.M. 1983. Note on the ecology of corticolous epiphyte dwellers 2. Collembola. Pedobiologia 25: 271-278.

Badejo, M.A., T.I. Nathaniel \& G. Tian. 1998. Abundance of springtails (Collembola) under four agroforestry tree species with contrasting litter quality. Biol. Fertil. Soils 27: 15-20.

Barrera, A. 1968. Distribución cliserial de los Siphonaptera del volcán Popocatépetl, su interpretación biogeográfica. A. Inst. Biol., serie Zool. 1: 35-100.

Behan-Pelletier, V. \& G. Newton. 1999. Linking soil biodiversity and ecosystem function - the taxonomic dilemma. BioScience 49: 149-153.

Cassagne, N., T. Gauquelin, M.C. Bal-Serin \& C. Gers. 2004. Endemic collembola, privileged bioindicators of forest management. Pedobiologia 50: 127-134.

Christiansen, K. 1992. Springtails. Kansas School Natur. 39: $1-16$.

Cutz-Pool, L.Q., J.G. Palacios-Vargas \& G. Castaño-Meneses. 2005. Diversidad y abundancia de colémbolos de musgos corticícolas en el Volcán Iztaccíhuatl (otoño 2003). Entomol. Mex. 4: 171-175.

Cutz-Pool, L.Q., A. García-Gómez \& A. Bernal-Rojas. 2006. Variación estacional de los invertebrados asociados a musgos corticícolas en la parte NW del Volcán Iztaccíhuatl, Estado de México, México. Entomol. Mex. 5: 227-231.

Cutz-Pool, L.Q., J.G. Palacios-Vargas \& G. Castaño-Meneses. 2008. Estructura de la comunidad de colémbolos (Hexapoda: Collembola) en musgos corticícolas en un gradiente altitudinal de un bosque templado subhúmedo. Rev. Biol. Trop. 56: 739-748.

Ducarme, X.H.M.A., G. Wauthy \& P. Lebrun. 2004. Are there real endogeic species in temperate forest mites? Pedobiologia 48: 139-147.

García-Gómez, A., G. Castaño-Meneses \& J.G. PalaciosVargas. 2009. Diversity of springtails (Hexapoda) in an altitudinal gradient in Iztaccíhuatl Volcano, Mexico. Pesq. Agrop. Bras. 44: 911-916.

Hawes, T.C., M.R. Worland, P. Covey \& J.S. Bale. 2007. Aerial dispersal of springtail on the Antarctic Peninsula: implications for local distribution and demography. Antarct. Sci. 19: 3-10.

Hasegawa, M., M.T. Ito \& K. Kitayama. 2006. Community structure of oribatid mites in relation to elevation and geology on the slope of Mount Kinabalu, Malaysia. Eur. J. Soil. Biol. 42: S191-S196.

Hou, P.C.L., X. Zou, C.Y. Huang \& H.J. Chien. 2005. Plant litter decomposition influenced by soil animals and disturbance in a subtropical rainforest of Taiwan. Pedobiologia 49: 539-547.

Heaney, L. 2001. Small mammal diversity along elevational gradients in the Philippines: an assessment of patterns and hypotheses. Global Ecol. Biogeogr. 10: 15-39.

Hopkin S.P. 1997. Biology of the springtails (Insecta: Collembola). Oxford, Oxford, Inglaterra.

Irmler, U. 2006. Climatic and litter fall effects on collembolan and oribatid mite species and communities in a beech Wood base don a 7 years investigation. Eur. J. Soil Biol. 42: 51-62.

Jensen, P., G.L. Jacobson \& D.E. Willard. 1973. Effects of moving and raking on collembola. Ecology 54: 564-572.

Jing, S., T. Solhøy, W. Huifu, T.I. Vollant \& X. Rumei. 2005. Differences in soil arthropods communities 
along a high altitude gradient at Shergyla Mountain, Tibet, China. Arctic. Alpin. Res. 37: 261-266.

Koleff, P., K.J. Gaston \& J.J. Lennon. 2003. Measuring beta diversity for presence-absence data. J. Anim. Ecol. 72: 367-382.

Linlden, D.R., P.F. Hendrix, D.C. Coleman \& P.C.J. van Vliet. 1994. Faunal indicators of soil quality, p. 91-106. In J.W. Doran, D.C. Coleman, D.F. Bezdicek \& B.A. Stewart (eds.). Defining Soil Quality for a Sustainable Environment. SSSA Special Publication No. 35, Soil Science Society of America Inc., American Society of Agronomy Inc., Madison, Wisconsin, EEUU.

Liu, W., J.E.D. Fox \& Z. Xiu. 2000. Leaf litter decomposition of canopy trees, bamboo and moss in a mountain moist evergreen broad-leaved forest on Ailao Mountain, Yunnan, South-west China. Ecol. Reserch. 15: 435-447.

Ludwing, J.A. \& J.F. Reynolds. 1988. Statistical ecology. John Wiley \& Son, USA.

Magurran, A.E. 1988. Ecological diversity and its measurement. Princeton University, New Jersey, EEUU.

Miranda A.R. \& J.G. Palacios-Vargas. 1992. Estudio comparativo de las comunidades de colémbolos edáficos de bosque de Abies religiosa y cultivo de haba (Vicia faba). Agrociencias Protec. veg. 3: 7-18.

O’Donnell, S. \& A. Kumar. 2006. Microclimatic factors associated with elevational changes in army ant density in tropical montane forest. Ecol. Entomol. 31: 491-498.

Palacios-Vargas, J.G. 1981. Collembola asociados a Tillandsia (Bromeliaceae) en el derrame lávico del Chichinautzin, Morelos, México Southwestern. Entomol. 6: 87-98.

Palacios-Vargas, J.G. 1985. Artrópodos del Popocatépetl. Tesis de Doctorado, Universidad Nacional Autónoma de México, México.

Palacios-Vargas, J.G. 1988. Consideraciones biogeográficas de los Microartrópodos del Popocatépetl, México. Folia Entomol. Mex. 75: 147-155.

Palacios-Vargas, J.G. \& G. Castaño-Meneses. 2002. Collembola associated with Tillandsia violacea (Bromeliaceae) in Mexican Quercus-Abies forest. Pedobiología 46: 395-403.

Pearson, D.L. 1977. A practical comparison of bird community structure on six lowland forest sites. The Condor 79: 232-244.
Pianka, E.R. 1966. Latitudinal gradients in species diversity: a review of concepts. Am. Nat. 910: 33-46.

Prinzing, A. 2005. Corticolous arthropods under climatic fluctuations: compensations is more important than migration. Ecology 28: 17-28.

Prinzing, A. \& S. Woas. 2003. Habitat use stratification of Collembola and oribatid mites, p. 271-281. In Y. Basset, V. Novotny, S.E. Miller \& R.L. Kitching (eds.). Arthropods of tropical forest: spatio-temporal dynamics and resource use in the canopy. Cambridge University, Reino Unido.

Rusek, J. 1998. Biodiversity of collembola and their functional role in the ecosistem. Biodivers. Conserv. 7: $1207-1219$.

Sariyildiz, T., J.M. Anderson \& M. Kucuk. 2005. Effects of tree species and topography on soil chemistry, litter quality, and decomposition in Northeast Turkey. Soil. Boil. Biochem. 37: 1695-1706.

Schowalter, T.D. 2006. Insect ecology, an ecosystem approach. Academic, London, Reino Unido.

Scout, N.A. \& D. Binkley. 1997. Foliage litter quality and annual net $\mathrm{N}$ mineralization: comparison across North American forest sites. Oecologia 111: 151-159.

Stevens, G.C. 1992. The elevational gradient in altitudinal range: an extension of Rapoport's latitudinal rule to altitude. Am. Nat. 140: 893-911.

Sokal, R.R. \& C.D. Michener. 1958. A statiscal method for evaluating systematic relationships. U. Kans. Sci. Bull. 38: 1409-1438.

Thaiutsa, B. \& O. Granger. 2000. El clima y la descomposición de la hojarasca en el bosque tropical. (Consultado: 4 mayo 2008, www.fao.org/docrep/n68455/ n68455s00.htm).

van Straalen, N. 1997. Community structure of soil arthropods as a bioindicator of soil health, p. 235-264. In C.E. Pankhurst, B.M. Doube \& V.V.S.R. Gupta (eds.). Biological indicators of Soil Health. CAB International, Wallingford, Reino Unido.

van Straalen, N. 1998. Evaluation of bioindicator systems derived from soil arthropod communities. Appl. Soil. Ecol. 9: 429-437.

Zar, J.H. 1984. Biostatystical Analysis. Prentice-Hall, Englewood Clif, Nueva Jersey, EEUU. 